\title{
Data Collection, Storage, and Retrieval with an Underwater Sensor Network
}

\author{
I. Vasilescu, K. Kotay, and D. Rus \\ MIT CSAIL \\ The Stata Center, 32 Vassar Street \\ Cambridge, MA 02139 \\ fiuliuv, kotay, rus g@csail.mit.edu
}

\author{
M. Dunbabin and P. Corke \\ CSIRO ICT Centre \\ 1 Technology Ct \\ Brisbane, Australia \\ f matthew.dunbabin, peter.corke g@csiro.au
}

\begin{abstract}
In this paper we present a novel platform for underwater sensor networks to be used for long-term monitoring of coral reefs and fisheries. The sensor network consists of static and mobile underwater sensor nodes. The nodes communicate point-to-point using a novel high-speed optical communication system integrated into the TinyOS stack, and they broadcast using an acoustic protocol integrated in the TinyOS stack. The nodes have a variety of sensing capabilities, including cameras, water temperature, and pressure. The mobile nodes can locate and hover above the static nodes for data muling, and they can perform network maintenance functions such as deployment, relocation, and recovery. In this paper we describe the hardware and software architecture of this underwater sensor network. We then describe the optical and acoustic networking protocols and present experimental networking and data collected in a pool, in rivers, and in the ocean. Finally, we describe our experiments with mobility for data muling in this network.
\end{abstract}

\section{Categories and Subject Descriptors}

C.2.4 [Computer-Communications Networks]: Distributed Systems; C.3 [Special-Purpose and ApplicationBased Systems]: Real-time and embedded systems

\section{General Terms}

Algorithms, Design, Experimentation

\section{Keywords}

mobile sensor networks, underwater networks, data muling

\section{INTRODUCTION}

The application of wireless sensor networks to the underwater domain has huge potential for monitoring the health of river and marine environments. The oceans alone cover $70 \%$

Permission to make digital or hard copies of all or part of this work for personal or classroom use is granted without fee provided that copies are not made or distributed for profit or commercial advantage and that copies bear this notice and the full citation on the first page. To copy otherwise, to republish, to post on servers or to redistribute to lists, requires prior specific permission and/or a fee.

SenSys'05, November 2-4, 2005, San Diego, California, USA.

Copyright 2005 ACM 1-59593-054-X/05/0011 ...\$5.00. of our planet and along with rivers and lakes are critical to our well-being. Monitoring these environments is difficult and costly for humans: divers are regulated in the hours and depths at which they can work, and require a boat on the surface that is costly to operate and subject to weather conditions. A sensor network deployed underwater could monitor physical variables such as water temperature and pressure as well as variables such as conductivity, turbidity and certain pollutants. The network could track plumes of silt due to dredging operations or pollutants flowing in from land, and it could monitor and model the behavior of underwater ecosystems. Imaging sensors could be used to measure visible change in the environment or count, and perhaps even classifyspecies.

However a number of problems confront us in achieving this goal. Some such as power efficiency, deployment and repair are common to wireless sensor network deployments on land, though more difficult in the underwater environment. Other issues render the problem radically different. A key issue is communications - current terrestrial wireless sensor network applications to date have used radio. At frequencies that are practical with low-cost radio chips and compact antennas, radio waves are attenuated so strongly in salt water that radio communications is impractical.

In this paper we describe an underwater sensor network system that consists of static and mobile sensor nodes (see Figure 1). The system is networked with two communication modalities: ultrasonic and optical. Ultrasonic communications has a long history for underwater applications and is widely used with autonomous underwater vehicles. It has many similarities to radio: it is a shared medium that supports broadcasting, but the low propagation speed ${ }^{1}$ poses a challenge for carrier-sense transmission strategies. Optical communication is capable of much higher data transmission rates and the propagation speed is much closer to the speed of light ${ }^{2}$. Unlike ultrasonic and radio communications, optical communication is essentially directional. This dual networking scheme enables many underwater sensor network applications, as it supports low-speed broadcast (e.g. necessary for localization) and high-speed directional data transfer (e.g. for monitoring).

An important characteristic of the underwater sensor network we built is mobility, whose benefits have been explored previously in $[6,15-17]$. The sensor network includes both

\footnotetext{
${ }^{1}$ Due to relatively low speed of sound in water of approximately $1500 \mathrm{~m} / \mathrm{s}$.

${ }^{2} v=c / n$ where $n$ is the refractive index of the medium.
} 
static and mobile nodes. Mobility enhances the performance of this sensor network in several ways. First, it provides a means for deploying, reconfiguring, and retrieving the nodes in the network. Second, it permits large area coverage with sparse networks which is especially important in an underwater environment - a much harder space to access than terrestrial space. The mobile nodes can move across the field to ensure the necessary connectivity. Third, mobile nodes can act as data mules and travel from node to node across a sparsely deployed sensor network to collect data. Communications is enabled only when the sensors and the mobile mules are in close proximity. Transmitting data over these shorter distances reduces the power consumption on each sensor and alleviates the hot spot problem on the sensors near the destination. Moreover, since underwater acoustic communication is characterized by low data rates, and optical underwater communication is subject to short ranges, mobility enables a time-efficient and more energy-efficient means to collect and transmit the data.

Specifically, we use the mobile nodes as data mules. Our data muling solution relies on a mobile node which is an autonomous underwater vehicle (AUV) with a matching optical communications link. This node can locate the static nodes using an optical location system. The AUV visits the nodes periodically to upload the data. The nodes are mostly in a deep sleep mode and wake every few seconds to determine if they are being optically signalled. This creates a desirable asymmmetry in the communications power required, where the AUV, which is mobile and rechargeable, takes on the energy expensive role. However we can introduce communication efficiencies on the AUV and only signal to an object that looks like a node. Our nodes are all in yellow watertight boxes that can be detected using a passive stereo vision system onboard the AUV, see Figure 3(c). The ultrasonic broadcast communications mode is used to notify the AUV about exceptional situtations.

We have deployed a network of 10 static nodes and two mobile nodes in two underwater environments: in deep pools and in the ocean. In this paper we report on the hardware for the static and mobile nodes, the optical and acoustic networking infrastructure we created for this system, our approach to data storage and retrieval, and the data muling system. We describe the system, its supporting algorithms, and experimental data collected in the pool, in a river, and in the ocean at Moreton Bay creek. This work is the result of a close collaboration between MIT and CSIRO.

\subsection{A Motivating Scenario: Data Collection}

The underwater sensor network we propose will facilitate the study of complex underwater systems by regulating and automating data collection. The static sensor nodes enable systematic recording of data. The mobile nodes enable efficient data muling and integration, data delivery to a surface base station independent of the physical location of the sensors, and long-term underwater operations of the sensor nodes at fixed locations.

Consider monitoring a large area of the sea floor where the nodes are placed on a $200 \mathrm{~m}$ grid and cover an area of $10 \times 10 \mathrm{~km}$ with $50 \times 50$ nodes. The total path length to visit all nodes in a raster fashion is $50 \mathrm{~km}$ which at an efficient submarine cruising speed of $0.5 \mathrm{~m} / \mathrm{s}$ would take nearly 28 hours of travel time. Further, each node is making a measurement every 10 minutes which comprises 4 bytes, giving a total data yield of 24 bytes/hour, or .56 kbytes/day. The $50 \times 50$ node network will store $1.37 \mathrm{Mbytes} /$ day. If the data from the network is uploaded every 5 days, the accumulated data for each upload will be 6.86 Mbytes.

The data could be collected in two ways: (1) with an autonomous robot functioning as a data mule using shortrange optical communication and (2) using an acoustic communication network with node-to-node routing. If the robot visited each node and used the optical transmission developed in this paper (whose data rate is 320 kbytes/sec) 6.86 Mbytes will be uploaded in 21 seconds and the total energy consumed will be $120 \mathrm{~J}$. This process will consume only $48 \mathrm{~mJ}$ from each node.

The energy per bit for acoustic modems is more difficult to obtain. The WHOI modem [2] has a data rate of $220 \mathrm{bits} / \mathrm{sec}$ over $5000 \mathrm{~m}$ at $10 \mathrm{~W}$ in transmition mode, or $20 \mathrm{~mJ} /$ bit. The Aquacomm modem has a data rate of $480 \mathrm{bit} / \mathrm{s}$ over $200 \mathrm{~m}$ at $0.45 \mathrm{~W}$, or $4.5 \mathrm{~mJ} /$ bit. Heidemann [11] anticipates $5 \mathrm{kbit} / \mathrm{s}$ over $500 \mathrm{~m}$ at $30 \mathrm{~mW}$ transmit power but does not provide the total power required or show experimental results. For this analysis we will assume $480 \mathrm{bit} / \mathrm{s}$ at $4.5 \mathrm{~mJ} / \mathrm{bit}$ with a range of $200 \mathrm{~m}$. Thus the $6.86 \mathrm{Mbytes}$ of data would require 1.3 days to transmit and the total energy consumed will be $247 \mathrm{~kJ}$. Because the modems have only $200 \mathrm{~m}$ range the data transfer will require multiple hops. If the average path length in the network is $5 \mathrm{~km}$ this will involve 25 hops, so the total energy consumed will be $6.2 \mathrm{MJ}$. In order to avoid collisions in the shared acoustic medium a sophisticated MAC strategy would be required. This strategy may also require a clock synchronization protocol.

The calculations are simplistic and ignore protocol and routing overhead. Nevertheless we can see that the energy consumption by the underwater network is over four orders of magnitude lower with the use of AUV data muling. If we further consider the cost of an optical communications board at $\$ 50 /$ node and the cost of the acoustic modem at $\$ 3000 /$ node, we argue that the most efficient way for collecting data from an underwater sensor network is using a system capable of optical communications with static and mobile nodes, such as the one described in this paper. The mobile nodes will require power to navigate the sensor network but they are easily rechargeable. The mobile node will maximize the lifetime and storage utilization for a fixedconfiguration underwater sensor network. We have created an asymmmetry in the communications power required, enabling very low power operations on the nodes that are difficult to access and have fixed energy reserves. By constrast, the AUV which is mobile and can be recharged at the end of each mission, takes on the energy expensive role.

\subsection{Related Work}

This work builds on a large body of research in designing and building underwater robots $[1,7,9,27,29]$ and sensor networks $[12,13]$. Much of the underwater work to date has been concerned with cabled networks [14] which require significant engineering and maintenance issues, and acoustic networking [3,11,22]. Kilfoyle [18] provides an excellent review of underwater acoustic communications. Some of the challenges with designing underwater sensor networks have been articulated in [3]. In [26] a low-power and low-cost optical communications system specifically for the task of submarine to sensor node is described. In [23] an optical sytem for communications and ranging is described. The 


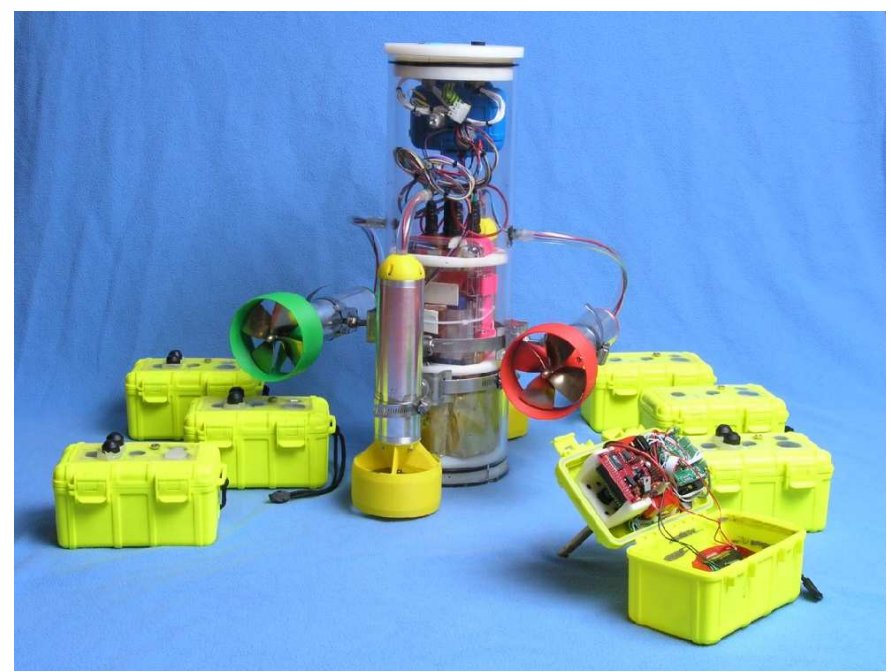

(a)

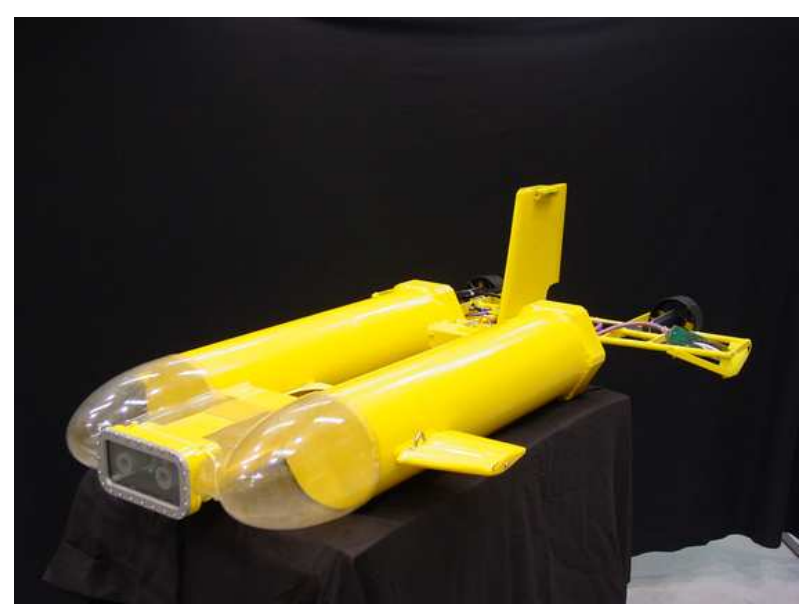

(b)

Figure 1: Group photo of the underwater sensor nodes. (a) the static sensor nodes (Aquaflecks) and a mobile node (Amour AUV). (b) a mobile node (Starbug AUV).

use of robots and sensor networks has been previously mentioned $[4,6,26]$. Recent results on using mobility in sensor networks to increase the network coverage and reduce power consumption suggest that data muling is an effective means of networking. In [24], Jain et al. proposed to use mobile mules, such as buses and animals to collect data by moving across a sparsely deployed sensor network. Communications is enabled only when the sensors and the mobile mules are in close proximity. Transmitting data over these shorter distances reduces the power consumption on each sensor. In [10] a study on how mobility can be used to increase the capacity of ad-hoc wireless network is presented. In [5,21] a study that analyzes performance in terms of an integrated measure of capacity, delay, and mobility is presented. In [28] an analysis of power consumption in sensor networks is presented.

\section{THE HARDWARE INFRASTRUCTURE}

Our research has two critical hardware elements: the static underwater sensor nodes, and the autonomous underwater vehicles (AUVs) which provide communications, mobility and a means of sensor node deployment and recovery. In our work we employed two AUVs: Starbug and Amour.

\subsection{Underwater Sensor Node Aquafleck}

We have built 20 underwater sensor nodes called Aquaflecks (see Figure 2). Each node is build around a CPU unit developed by CSIRO called a Fleck [25], based on the ATmega128 processor, with $128 \mathrm{kbyte}$ of program flash memory, $4 \mathrm{kbyte}$ of RAM, and 512kbyte of flash memory for data logging/storage. The Fleck is interfaced to a special optical communications board through 2 digital IO pins. One of these pins is used to turn an LED on or off, while the other is used to sense the output from a matched photodiode. All the analog electronics (e.g., amplifiers etc) are on the interface board. The Fleck is also interfaced with a sensor board. The boards are connected in a stack using stack-through connectors.
The underwater sensor node is contained in a yellow watertight Otter box that measures $170 \times 100 \times 90 \mathrm{~mm}$ and has been modified to incorporate the sensing and communication hardware. The Otter box is guaranteed to be watertight up to a depth of 30 meters. Each box has a high speed optical communication module that uses $532 \mathrm{~nm}$ light, and is capable of a range of $2.2 \mathrm{~m} / 8 \mathrm{~m}^{3}$, within a cone of 30 degrees and a maximum data rate of $320 \mathrm{kbits} / \mathrm{s}$. Additionally, there is a acoustic communication module using $30 \mathrm{kHz}$ FSK modulation with a range of $20 \mathrm{~m}$ omnidirectional, and a data rate of $50 \mathrm{bit} / \mathrm{s}$. The same module is also used for ranging ${ }^{4}$. For sensing, each node has a pressure sensor, temperature sensor, and a CMUCam camera capable of color pictures with a $255 \times 143$ resolution. The top side of the box contains a 170 $\mathrm{mm}$ rod with an LED beacon. The rod can be used by an AUV to locate the box, dock, and pick it up. Future versions will contain a XENON flash tube for increasing the distance for reliable node location to about 20 meters. The sensor node is powered by 3 alkaline $\mathrm{C}$ cells. Three $\mathrm{C}$ cells can provide 27 wh and four days of continuous operation with all sensors and communication hardware fully powered. The box is weighted to be $40 \%$ negatively buoyant, and balanced such that if dropped in water it always lands top up.

\subsection{Amour AUV}

In this project Amour is a mobile node AUV used to dock and transport the Aquafleck nodes. Amour can also locate an underwater sensor node and hover above it for data muling. Figure 1(Top) shows Amour next to Aquaflecks.

The robot's body consists of an acrylic tube that is 48.26 $\mathrm{cm}$ long and $15.24 \mathrm{~cm}$ in diameter. It has four external thrusters with a maximum power of $150 \mathrm{~W}$ and a maximum static thrust of $35 \mathrm{~N}$ each. The robot is statically balanced in an upright position. Two of its thrusters are aligned vertically. A pressure sensor provides depth feedback. The other

\footnotetext{
${ }^{3}$ The $8 \mathrm{~m}$ range requires lenses on one of the devices and actively pointing it toward to other

${ }^{4}$ Two sensor nodes can determine the distance between them using time of flight of the sound waves
} 


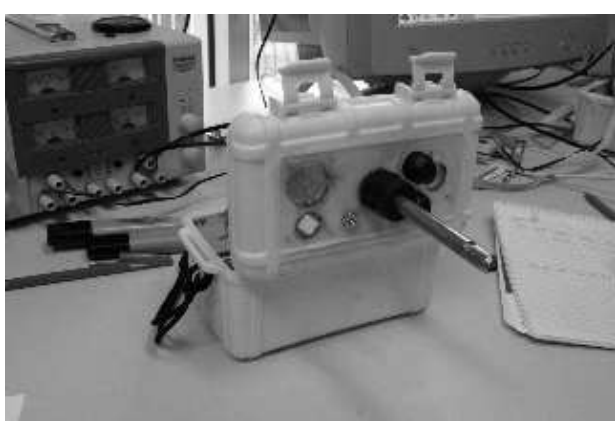

(a)

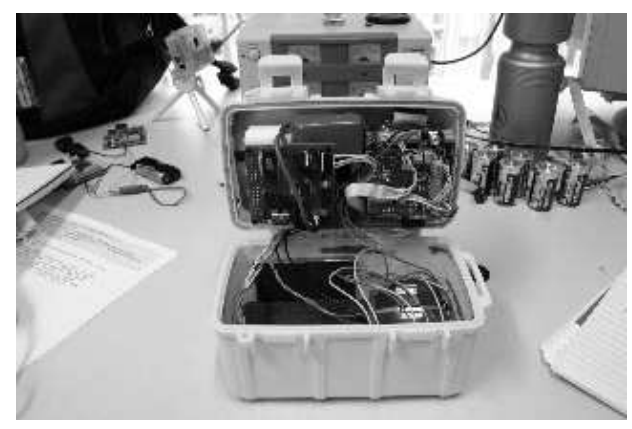

(b)

Figure 2: The underwater sensor node. (a) top of the node containing the sensors and the docking rod, (b) inside showing circuitry.

two thrusters are positioned horizontally to provide forwardbackward movement in the horizontal plane as well as rotation. A magnetic compass is used for orientation feedback and enables patterns of navigation, for example movement along a grid and spiral search. The power is provided by a $140 \mathrm{Wh}$ lithium polymer battery. The main processor is a 8-bit microcontroller with 64kbyte of program memory and $2 \mathrm{kbyte}$ of RAM. The bottom cap of the robot has a cone shaped cavity, designed for maximum mechanical reliability in docking and for optical communication. The robot can dock with sensor nodes in order to pick them up and transport them to a new location. This operation enables autonomous network deployment, reconfiguration, and retrieval. The docking system is general in that the robot can dock with any mate whose docking element is a $15.24 \mathrm{~cm}$ long rod of $1 \mathrm{~cm}$ diameter. The bottom cap also contains 4 light sensors pointing in complementary directions. The sensors can determine the direction toward a high frequency modulated light source (an LED) from up to 8 meters in clear water. A latching mechanism can hold the docked element with up to $200 \mathrm{~N}$ of force. The robot includes the same optical communication units as the static sensor nodes described in Section 2.1. Most of the electronics inside the robot, including the batteries, are placed in small Otter watertight cases. Recharging the batteries or reprogramming the robot can be done through watertight top cap connectors, without opening the main body.

\subsection{Starbug AUV}

In this work Starbug is a mobile node used to locate the Aquafleck nodes by vision, to hover above Aquaflecks for data muling, and to dock with Amour in order to provide visual control feedback for long range navigation. This form of coordinated control is the subject of another paper.

Starbug is a hybrid AUV designed to optimize endurance, manoeuvrability and functionality [7]. Endurance is best achieved with a streamlined torpedo style vehicle, however, this requires the vehicle to have longitudinal motion to obtain any control authority. Manoeuvrability is best achieved with the well actuated "crate" style vehicles typical of most research platforms. These generally have control authority in multiple directions to allow station keeping although they are power hungry and consequently usually tethered. Both these style of vehicles have limited functionality away from research purposes. The "Starbug" vehicle is a hybrid of these two concepts with extra design features added to in- crease the functionality of the platform through provisions for manipulators and scientific payloads.

The key performance specifications for Starbug are: mass $26 \mathrm{~kg}$, length $1.2 \mathrm{~m}$ (folding to $0.8 \mathrm{~m}$ for transport), maximum forward thrust $20 \mathrm{~N}$, maximum speed $1.5 \mathrm{~m} / \mathrm{s}$, Speed for maximum range $0.7 \mathrm{~m} / \mathrm{s}$, hotel load $1.1 \mathrm{Amps}$, and battery capacity $6.4 \mathrm{Ah}(4 \mathrm{x} 12 \mathrm{~V}$ sealed lead acid batteries). The vehicle is fully actuated with six thrusters providing forward, lateral and vertical translations as well as yaw, roll and pitch rotations. Vehicle control software effectively decouples the thruster force and allows independent control of vehicle force and moment in $6 \mathrm{DOF}$. All the thrusters are daisychained on a CANBus control network, allowing for a single hull penetration. Internal sensors such as pressure and IMU are also on the CANbus.

Starbug has two stereo vision heads. One looking downward for sea-floor altitude and speed estimatation as well as mapping, and the other looking forward for obstacle avoidance. The camera pairs have a baseline of $70 \mathrm{~mm}$. This allows an effective height resolution in the range 0.2 to $1.7 \mathrm{~m}$. All cameras are tightly synchronized and line multiplexed into PAL format composite video signal. A $3 \mathrm{~W}$ white LED located in each camera housing provides additional scene lighting. The onboard computer is a PC/104 stack with a Crusoe processor running Linux. An Aquacomm acoustic communications link with a bandwidth of $100 \mathrm{bps}$ and range of $200 \mathrm{~m}$ is also fitted.

\section{NETWORKING}

Sensor network communication on land is primarily radiobased, due to the relatively low power needed to transmit radio messages and the basically omnidirectional nature of radio propagation. Unfortunately, the majority of the electromagnetic spectrum is significantly attenuated by saltwater, rendering radio communication useless for this application. The exception is in the visible light portion of the spectrum that is less attenuated, especially in the the frequency of blue-green light. The primary advantage of optical communication is the higher theoretical data rate due to the higher frequency signal, while the disadvantages are range and line-of-sight operation.

The other obvious approach for underwater communication is sound. This has been used extensively for localization (SONAR) and short range communication (the "gertrude" or UQC underwater telephone). While acoustic communication can be used for much longer range communication 


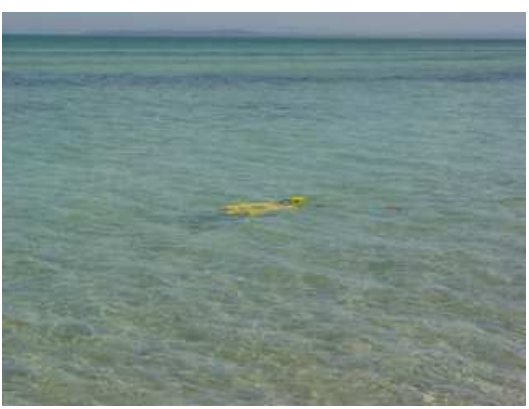

(a)

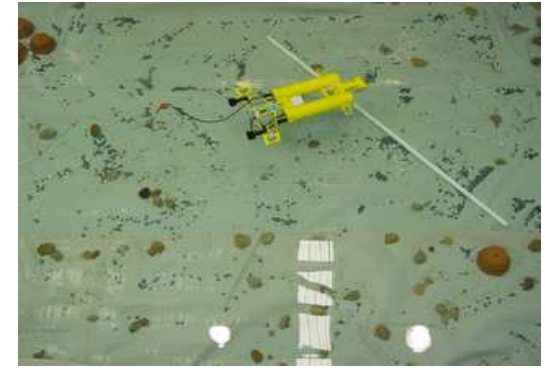

(b)

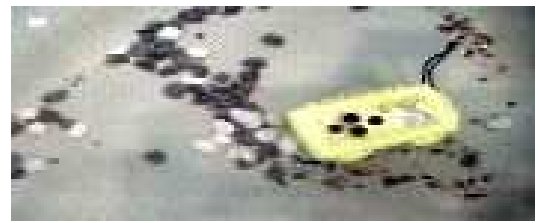

(c)

Figure 3: (a) Starbug in Moreton Bay, Brisbane. (b) Starbug in the pool. (c) Image of Aquafleck at bottom of the pool, as taken from the Starbug AUV and used for node identification and relative positioning.

than optical it also suffers from attenuation, with higher frequencies being attenuated more than lower frequency signals. Thus a tradeoff is required between communication range and data rate. Other issues that affect acoustic communication are reflections from the environment: the water surface, the bottom, and underwater structures such as reefs or geological features. A significant advantage for employing acoustic communication in an underwater sensor network is the ability to use acoustic signals for sensor node localization. The speed of sound in water is low enough to permit accurate timing of signals to determine the distance between nodes. Pairwise node distance data can then be used to perform 3D localization, similar to the 2D localization demonstrated in [20]. Underwater acoustic localization methods [19] include long baseline (LBL) and ultra-short baseline (USBL) approaches.

Our approach to communication is based on a hybrid design that incorporates both optical and acoustic systems. The optical system is used for short-range line-of-sight data transfer and communication between a sensor node and an AUV acting as a data mule. The AUV moves through the network downloading the stored data from the sensor nodes, and uploading commands. The acoustic system is used to signal events and transmit small amounts of data. Signalling an event allows the AUV to move to the area of interest, and may trigger a redeployment of the sensor network to concentrate on some important feature in the environment. The optical and acoustic systems are described below.

\subsection{Optical Communication}

Optical communication, while restricted to short-range line-of-sight applications has a much higher theoretical bandwidth than acoustic communication. This makes it appealing when large amounts of data need to be transferred. In a long-term underwater sensing situation, each sensor node may accumulate a sizeable amount of data, especially if the nodes are equipped with cameras. In our current sensor node design, each node can accumulate up to $512 \mathrm{kbyte}$ of data. Sending this amount of data over a low-speed acoustic communication link would require a significant amount of time, and would also prevent other nodes from communicating acoustically during the transfer due to the broadcast nature of acoustic communication. However, using optical communication, the data can be transferred quickly without preventing event signalling using acoustic communication.

Optical communication range is affected by factors such as the light absorption of water, scattering, beam divergence, and ambient light. The absorption is described by

$$
I_{1}=I_{0} \times e^{-k\left(d_{1}-d_{0}\right)},
$$

where $I_{0}$ is the intensity at distance $d_{0}$ from the source, $I_{1}$ is the intensity at distance $d_{1}$ from the source, and $k$ is the absorption coefficient that depends on frequency. Example absorption coefficients are shown in Table 1. Turbidity, the clarity of water due to suspended particles, also affects range. Turbidity absorption is a more complex attenuation that is frequency independent and is not well modelled. There is also a quadratic attenuation of light,

$$
I_{1}=I_{0} \times\left(d_{0} / d_{1}\right)^{2},
$$

such that doubling the distance results in $1 / 4$ of received power. Ambient light can saturate the sensitive receiver if the system is utilized near the surface of the water. To prevent this we install an optical filter as described below.

\subsubsection{Hardware}

The optical transmitter is the Luxeon 5 LXHL-PM02, a $532 \mathrm{~nm}$ (green) LED with approx. $700 \mathrm{~mW}$ radiated power while consuming $6 \mathrm{~W}$ of input power (approx. 10-15\% efficiency). The high power rating permits it to be used in a low duty cycle mode, allowing an intense pulse of light to be generated for a short period of time. The receiver is a PDB-C156 high speed PIN photodiode with $8 \mathrm{~mm}^{2}$ surface area. The choice of the light frequency is based on the sensitivity of the photodiode and the attenuation of light as given by Equation 1. The sensitivity of the photodiode is shown in Table 1. The photodiode is more sensitive to the infrared and red wavelengths than to green, but green is less absorbed by water.

The output current is proportional to

$$
S \times e^{-k\left(d_{1}-d_{0}\right)} \times P,
$$

where $\mathrm{S}$ is sensitivity and $\mathrm{P}$ is the power in watts. Figure 4 shows how the output current varies according to distance for different wavelengths of light. Due to the severe attenuation of infrared in water, the output current at $0.2 \mathrm{~m}$ is less than for green light at $10 \mathrm{~m}$. Note that red light outperforms green light up to $0.5 \mathrm{~m}$ but green is better at $1 \mathrm{~m}$ and beyond. Thus we have chosen to use green light for our optical communication system. 


\begin{tabular}{|l|l|l|}
\hline & $\begin{array}{l}\text { Photodiode } \\
\text { Sensitivity }\end{array}$ & $\begin{array}{l}\text { Absorbtion } \\
\text { Coefficient }\end{array}$ \\
\hline \hline 532nm (green) & $0.39 \mathrm{~A} / \mathrm{W}$ & $3.2 \times 10^{-4} \mathrm{~cm}^{-1}$ \\
650nm (red) & $0.45 \mathrm{~A} / \mathrm{W}$ & $3.2 \times 10^{-3} \mathrm{~cm}^{-1}$ \\
890nm (infrared) & $0.65 \mathrm{~A} / \mathrm{W}$ & $6.0 \times 10^{-2} \mathrm{~cm}^{-1}$ \\
\hline
\end{tabular}

Table 1: Sensitivity for PDB-C156 High speed PIN photodiode and adsorption coefficients in water for different wavelengths of light.

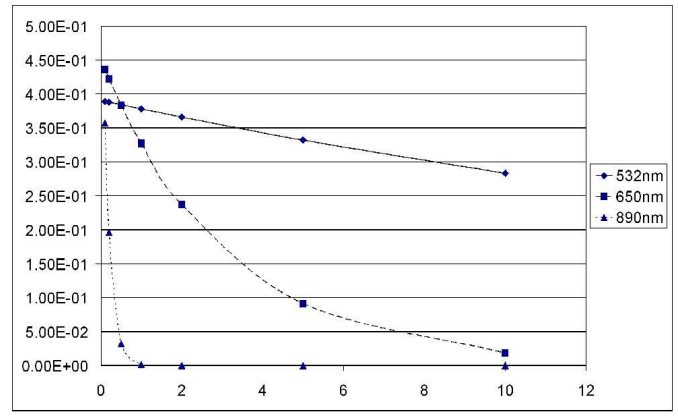

Figure 4: Calculated photodiode output current values at various distances.

To improve the effectiveness of the optical system we use a Fraen acrylic concentrator on the LED to create a cone of light with an internal angle of 30 degrees. This increases distance at the expense of transmission angle. The LED is switched by a high speed MOS transistor with low input capacitance in order to achieve sharp turn-on edges, that can be easily detected by the receiver.

We also use a green dichroic filter on the photodiode to attenuate frequencies other than green - the sun would otherwise saturate the receiver in shallow water. The filter is the same type used in video cameras to filter light going to the green CCD (the other two filters separate the red and blue wavelengths). It has a $90 \%$ transmissivity in the $500-$ $550 \mathrm{~nm}$ band, with less than $5 \%$ in the rest of the spectrum. The photodiode signal (current) is amplified and converted into a voltage signal and an automatic gain control circuit maintains the amplitude of the signal which is converted to a digital format and fed into a microcontroller input.

\subsubsection{Protocol}

Several optical communication formats have been developed in conjunction with the IRDA protocol, which is used for short-range optical communication between computer devices. Examples are NRZ (non-return to zero), SIR, FIR, and VFIR (also known as 4PPM). NRZ is the common RS232 asynchronous serial communication format where data bits of ' 0 ' are sent with a full bit-time high signal and '1' values are send with a low signal. SIR and FIR are extensions of NRZ with a shortened high-time for power conservation. Each of these is an example of pulse width modulation, while VFIR uses pulse position modulation. In this system there are four discrete possible pulse positions, and the position of the pulse is determined by the value of two bits of data. This format requires a preamble to synchronize the pulse timing of the message. We have adapted this system by using a start pulse for each byte, followed by a pulse whose

\begin{tabular}{|l|l|}
\hline State & Time Interval \\
\hline \hline Data bits 00 & 4.0 us \\
\hline Data bits 01 & 4.5 us \\
\hline Data bits 10 & 5.0 us \\
\hline Data bits 11 & 5.5 us \\
\hline End of byte & $6.0 \mathrm{us}$ \\
\hline End of packet & $10.0 \mathrm{us}$ \\
\hline
\end{tabular}

Table 2: Time intervals between high value pulses in our modified VFIR optical communication format.

interval from the start pulse is determined by the value of the first two data bits. The next two data bits then determine the offset from the previous pulse, and so on. Finally, the interval to the start pulse of the next byte and the interval between packets have a specific time intervals. These intervals are given in Table 2.

Each pulse is 250ns wide-it must be greater than the minimum turn on time of the LED, 100ns, but should be as short as possible to minimize power consumption. $250 \mathrm{~ns}$ is the shortest pulse that can be created in software from an I/O pin of the Fleck microcontroller. This results in an average of $(4+4.5+5+5.5+6) / 8=3.125$ us per bit or $320 \mathrm{kbit} / \mathrm{s}$. No other hardware overhead is required. The delays between the bits were dictated by the ability of our software to distinguish and decode the bits on $8 \mathrm{MHz}$ processor in the Fleck. One of the advantages of this encoding is that the receiver gets a continuous sequence of pulses regardless of the data (as opposed to NRZ and its variants), and can be easily kept in sync. Smaller delays can be implemented if a hardware decoder or a faster processor is used.

Packets have the same format as TinyOS packets. Only one preamble byte is used (0x0f) - since our encoding has synchronization at every byte the preamble is only required to determine possible packet beginnings and to prevent the incorrect reception of the first real data packet due to the special processing. A preamble byte is necessary to allow the microprocessor to concentrate on communication processing. The photodiode amplifier is connected to an interruptenabled I/O port on the microprocessor. When the first impulse is received the interrupt handler is called with an unknown latency that prevents accurate interval determination, possibly corrupting the first two data bits. Thus the preamble byte is discarded, and the microprocessor then disables interrupts and uses polled I/O to accurately measure the pulse intervals for all remaining packet bytes. Bytes are stored as received and the packet is verified by a CRC checksum.

\subsubsection{Experimental Characterization}

We have tested the optical system to determine the maximum communication range and the coverage area. Maximum communication range is the furthest distance at which complete packets are reliably received. ${ }^{5}$ The testing procedure consisted of placing a transmitting sensor node and a receiving sensor node in the water such that the top side

\footnotetext{
${ }^{5}$ In this case, the reception reliability is an observation that the receiver was flashing at a regular rate which matched the packet transmission rate - approximately one packet per second. However, it is possible that the reception rate was less than $100 \%$.
} 


\begin{tabular}{|l|l|l|l|}
\hline Distance & $\begin{array}{l}\text { Received } \\
\text { Packets }\end{array}$ & $\begin{array}{l}\text { Missed } \\
\text { Packets }\end{array}$ & $\begin{array}{l}\text { Packet Success } \\
\text { Rate }\end{array}$ \\
\hline \hline $2.1 \mathrm{~m}$ & 199 & 0 & $100.0 \%$ \\
$4.3 \mathrm{~m}$ & 199 & 0 & $100.0 \%$ \\
$5.3 \mathrm{~m}$ & 199 & 2 & $99.0 \%$ \\
$6.4 \mathrm{~m}$ & 199 & 0 & $100.0 \%$ \\
$7.0 \mathrm{~m}$ & 199 & 8 & $96.0 \%$ \\
\hline
\end{tabular}

Table 3: Reception rates for optical communication in clear water with a compound lens used to concentrate the light beam. Each experiment consisted of transmitting 199 packets with increasing ID numbers in each packet. The receiver recorded the number of packets received (maximum ID number - minimum ID number +1 ) and the number of missed packets (packets not received within the packet ID reception range). Experiments that have small packet ID reception ranges are the result of pointing errors during the experiment.

of the sensor node enclosures (where the LED and photodiode are mounted) were facing one another. Both boxes were at a depth of $30-40 \mathrm{~cm}$. The transmitter would then send packets at a rate of $1 \mathrm{~Hz}$ which, if received correctly, would illuminate an LED on the receiving node.

In clear water (an indoor swimming pool), the maximum range is $8 \mathrm{~m}$, in highly turbid water with a maximum visibility of $1.4 \mathrm{~m}$ (the Charles River) the maximum range is $1 \mathrm{~m}$. The coverage area is a $2 \Theta$ angle of 30 degrees.

In order to demonstrate longer range communication, a $60 \mathrm{~mm} \mathrm{~F} 1.0^{6}$ compound lens was added to the optical transmitter. This concentrates the light into a narrower cone, increasing the communication distance and Table 3 gives the experimental results. At up to $7 \mathrm{~m}$ it was possible to still obtain $96 \%$ reception if the transmitter was pointed directly at the receiver. Since the transmitter was held in place by hand against the pool wall, it is possible that it was moved slightly during the experiment. As the distance increases, the effect of a slight deviation is magnified.

A consequence of the narrower beam is less coverage which requires accurate pointing of the transmitter such that the beam falls on the receiver's photodiode. It may be difficult for an AUV to maintain accurate pointing of a powerful fixed lens onto a sensor node using only its thrusters in the presence of currents or waves. At $7 \mathrm{~m}$ distance even a small angular deviation could cause the beam to move away from the sensor node. Therefore a separate, higher bandwidth pointing assembly may be required to maintain a "lock" on the sensor node while the AUV thrusters are activated to keep the robot on station. Note that only the AUV would require the active pointing mechanism.

The energy expenditure of optical communication has been measured at $1094 \mathrm{~nJ} /$ bit, according to:

$$
E=P \times t_{\text {pulse }} \times 5 / 8,
$$

with $P$ the input power to the LED $(7 \mathrm{~W}), t_{\text {pulse }}$ the time per pulse (250ns), and using 5 pulses per 8 bits as described in Section 3.1.2. This compares favorably with Berkeley Mica moties which uses $760 \mathrm{~nJ} /$ bit transmitted [13].

\footnotetext{
${ }^{6}$ Measured in water.
}

\subsection{Acoustic Communication}

Our goal for acoustic communication is a system that can be used for event signalling, for low-bandwidth communication, and for 3D localization of the nodes in a sensor network. Event signalling and localization require very short acoustic emissions, possibly only a single ping if the devices can infer the identity of the transmitter. ${ }^{7}$ Otherwise several bits may be needed in the message to identify the sender, and possibly the intended recipient. Short messages have a greater likelihood of being correctly received, as reflections are less likely to arrive during reception of the message. In contrast, the latter bits of a long message may be plagued by multipath reflections from the early bits. Thus there is an emphasis on keeping the message length as short as possible.

\subsubsection{Hardware}

Underwater acoustic communications is a mature field [18, 22 . However, there are no inexpensive off-the-shelf acoustic modems that would fit in the small form factor of the Aquafleck. In this section we describe the acoustic communications system we developed for broadcast communication that supports event signaling and localization and is small and inexpensive so that it can be incorporated in each sensor node. The main trade-off is in distance; the range of our system is $25 \mathrm{~m}$ as compared to the range of the commercial systems which is on the order of kilometers [2]. For underwater applications focused around coral reefs a $25 \mathrm{~m}$ broadcast range is sufficient.

Transducers normally designed for air do not work well underwater due to the impedance mismatch as well as water sealing problems. However, we investigated the Panasonic EFR-RQB40K5 (receiver) and EFR-TQB40K5 (transmitter) which are completely sealed transducers rated as weatherproof for air use at $40 \mathrm{kHz}$. These units cost approx. $\$ 20$. We found that is is possible to use these devices in water if they are properly sealed (we use hot glue to attach them to the top of the Aquaflecks). When driven with a $30-32 \mathrm{kHz}$ signal, the acoustic coupling with water is very good and they emit uniformly over a hemisphere.

The transmitted signal is generated by an XR2206 integrated circuit, capable of generating two different frequencies on command, enabling the use of both amplitude modulation (ASK) as well as frequency shift keying (FSK) modulation. The signal is amplified to $20 \mathrm{~V}$ peak-to-peak before being applied to the transducer. The emitting power can be controlled, a feature mainly used for reducing the multipath reflections. On the receiving side the signal is amplified (40dB) and fed into both a PLL loop for decoding the FSK modulation and an adaptive ASK circuit, the latter is used mainly for detecting pulses for ranging.

\subsubsection{Experimental Characterization}

We have tested the acoustic communication system in an indoor swimming pool and in the Charles River. Experiments were conducted to determine the maximum range at which a square wave signal (an alternating data sequence of 0's and 1's) could be correctly received. The testing procedure consisted of placing a transmitting sensor node and a receiving sensor node in the water such that the top side of the sensor node enclosures (where the transducers are mounted) were facing one another. Both boxes were at

\footnotetext{
${ }^{7} \mathrm{~A}$ "ping" is a single output pulse of a sonar system.
} 


\begin{tabular}{|l|l|l|}
\hline Distance & $\begin{array}{l}\text { Missed } \\
\text { Packets }\end{array}$ & $\begin{array}{l}\text { Packet Success } \\
\text { Rate }\end{array}$ \\
\hline \hline $1 \mathrm{~m}$ & 2 & $96 \%$ \\
$2 \mathrm{~m}$ & 0 & $100 \%$ \\
$4 \mathrm{~m}$ & 4 & $92 \%$ \\
$6 \mathrm{~m}$ & 8 & $84 \%$ \\
$8 \mathrm{~m}$ & 13 & $74 \%$ \\
$10 \mathrm{~m}$ & 16 & $68 \%$ \\
$15 \mathrm{~m}$ & 22 & $56 \%$ \\
\hline
\end{tabular}

Table 4: Reception rates for acoustic communication in a tow tank. Each experiment consisted of transmitting 50 packets, with the receiver recording the number of missed packets. The data rate was $41 \mathrm{bit} / \mathrm{s}$.

\begin{tabular}{|l|l|l|l|}
\hline Distance & $\begin{array}{l}\text { Elapsed } \\
\text { Time }\end{array}$ & $\begin{array}{l}\text { Estimated } \\
\text { Distance }\end{array}$ & $\begin{array}{l}\text { Error } \\
\text { Percentage }\end{array}$ \\
\hline \hline $1 \mathrm{~m}$ & $1.4 \mathrm{~ms}$ & $1.04 \mathrm{~m}$ & $+3.7 \%$ \\
$2 \mathrm{~m}$ & $2.78 \mathrm{~ms}$ & $2.06 \mathrm{~m}$ & $+3.0 \%$ \\
$4 \mathrm{~m}$ & $5.55 \mathrm{~ms}$ & $4.11 \mathrm{~m}$ & $+2.8 \%$ \\
$6 \mathrm{~m}$ & $8.21 \mathrm{~ms}$ & $6.08 \mathrm{~m}$ & $+1.4 \%$ \\
$8 \mathrm{~m}$ & $11.1 \mathrm{~ms}$ & $8.23 \mathrm{~m}$ & $+2.8 \%$ \\
\hline
\end{tabular}

Table 5: Results of ranging experiments in a tow tank. The elapsed time represents twice the distance between the nodes. A speed of $1482 \mathrm{~m} / \mathrm{s}$ was used to estimate the distance.

a depth of $30-40 \mathrm{~cm}$. The transmitter would then continuously send the square wave signal and the reconstructed data stream output of the receiver was viewed on an oscilloscope or indicated by a flashing LED on the receiver. The pool tests were performed with a $1 \mathrm{~Hz}$ square wave data signal and the flashing LED output of the receiver at $10 \mathrm{~m}$ was visually consistent with the input signal, although it is possible some glitches may have occurred. At the river, the data rate was $50 \mathrm{~Hz}$ and at $5.8 \mathrm{~m}$ a square wave output was observed on the oscilloscope with no glitches. At $8.6 \mathrm{~m}$ glitches were apparent in the output, although communication would still be possible at a reduced data rate.

We also tested acoustic packet communication using a pulse position modulation (PPM) encoding to avoid problems with reflections. The packet size was 20 bytes and was checksummed to verify correct reception. The results are presented in Table 4. The data shows that acoustic communication is usable up to a distance of $15 \mathrm{~m}$ at a data rate of $41 \mathrm{bit} / \mathrm{s}$. The limitation on data rate was due to reflections in the tow tank, which were a problem even with the PPM encoding (the FSK encoding did not work at all with the severity of reflections in the tow tank). In open water we expect to achieve a higher data rate because the pulse period can be reduced. With more powerful transducers we can achieve increased communication range.

We have also conducted ranging experiments between two Aquaflecks using time or flight. The results are shown in Table 5. There is a consistent overestimation of the actual distance, possibly due to an inaccurate estimation of the speed of sound in the tow tank water. However, the error is under $4 \%$ which is suitable for use in our localiza- tion algorithm [20]. We are now implementing this network localization for the underwater system.

A localized underwater network with bimodal communication (optical for directional communication and acoustic for broadcast and ranging) has many interesting and useful applications. The network can localize and track the mobile nodes, essentially providing navigation beacons. The network can also be used to monitor and relay special events, to aggregate global perception maps, and to track and predict the change of events, for example the movement of fish or the spread of pollutants. We plan to build on this work and pursue these types of application with larger networks deployed in coral reefs.

\section{DATA COLLECTION}

The amount of data that can be collected over an extended period of time is dependent on the maximum data storage capacity of a sensor node and the battery lifetime. If data is collected at a rate that exceeds the storage capacity of the node during the expected battery lifetime of the unit, then the node must be retrieved prior to full battery expenditure or the data must be transmitted to another location. In the latter case, the energy cost of transmitting the data must be taken into account when determining the deployment time of sensor nodes.

Our sensor nodes have 512kbyte of flash memory in which to store collected data from the various sensors. If a node is equipped with a camera, then the memory may be filled quite quickly even if photographs are taken at a slow rate (a single $255 \times 143$ image requires 36465 bytes of data storage, assuming no compression.) Thus, in order to keep the sensor nodes on station as long as possible it is necessary to employ an AUV to periodically visit the nodes to download the stored data. This process if referred to as "data muling" and is described in Section 5 .

The energy required for data transfer is $1094 \mathrm{~nJ}$ per bit (see Section 3.1.3). Therefore the energy required to transfer the entire contents of the flash is 4.59J. The maximum rate of data transfer is $320 \mathrm{kbit} / \mathrm{s}$, resulting in a transfer time of 13.1 seconds. assuming some missed packets we will use a $100 \mathrm{kbit} / \mathrm{s}$ data rate. The power expended is $0.350 \mathrm{~W}$ while transmitting. The current consumption of the node itself is approximately $75 \mathrm{~mA}$ continuous. This reflects the $45 \mathrm{~mA}$ consumed by the Fleck, plus an additional $30 \mathrm{~mA}$ consumed by the optical receiver and the pressure and temperature sensors. It is possible to disable the optical receiver and sensors in order to conserve power. The Fleck can also enter a sleep state in which it consumes only $2 \mathrm{~mA}$. Power is supplied from three $\mathrm{C}$ cells in series, producing $4.5 \mathrm{~V}$ that is converted to $5 \mathrm{~V}$ and $7 \mathrm{~V}$ supplies by a $\mathrm{DC} / \mathrm{DC}$ converter. However, the cell voltage decreases as the cell is discharged so an average value of $1.2 \mathrm{~V}$ will be used for the following calculations. Given that a $\mathrm{C}$ cell has a nominal capacity of $7800 \mathrm{mAh}$, the energy in a cell is $33 \mathrm{~kJ}$ and the energy of the 3 -cell battery is $101 \mathrm{~kJ}$. The battery lifetime of the robot in days, $\mathrm{L}$, is then given by

$$
L=E_{\text {battery }} /\left(P_{c}+\left(P_{t} \times k\right)\right) /(60 * 60 * 24),
$$

where $P_{c}$ is the continuous power consumption, $P_{t}$ is the power expended when transmitting, and $k$ is the transmission duty cycle. For continuous transmission the lifetime is two days, but for transmitting once every 15 minutes the 
lifetime is extended to approximately four days. If a unit is in full sleep mode, the lifetime is 162 days. By using sleep mode judiciously, a node can be expected to function for several weeks while still accumulating data.

\subsection{Experiments}

We have done two types of data collection experiments: long term collection of temperature and pressure data in a creek affected by ocean tide, and large-scale data collection and storage with cameras.

Three sensor nodes were deployed in Tingalpa Creek, Brisbane and tasked with logging water temperature and water pressure. The sensor nodes were deployed approximately one kilometer apart along the creek for a period of three days. A file system recently developed at CSIRO was used to save data to the on-board flash memory. The nodes were programmed to log water pressure and temperature every 150 seconds. The file system was initialized such that it could hold seven days worth of data. Approximately $110 \mathrm{kbyte}$ of the $512 \mathrm{kbyte}$ on-board flash memory was used.

After the sensor nodes were retrieved, the logged data was uploaded via the optical-based communications link, which took around 388s to complete. Figure 5(a) and (b) shows the data acquired from the pressure sensor along with the high and low tide times, ${ }^{8}$ which are marked with broken lines. We can see an approximately one hour lag between the expected and observed low tide times. We understand that this is due to the dynamics of the water flowing in and out of a creek from the ocean.

We have also collected, stored, and retrieved images taken with the camera in a suite of experiments. Figure 5(c) shows an example of such an image. In Section 5 we provide the data transmission statistics for this type of data.

\section{DATA RETRIEVAL USING MOBILITY}

Data retrieval from a deployed network of Aquaflecks is done optically using the mobile nodes Starbug and Amour. Given a model for the deployed Aquaflecks, the task of the mobile node is to establish a tour of the network, locate each node in the tour one at a time, and hover above each node to download the data optically. During this period of communication the mobile node may also upload data to the static node, for example to adjust its clock or to change the data sampling rate. The key challenges for underwater data muling are (1) locating the first node; (2) locating the next node in the sequence; (3) controlling the hover mode for the mobile node; (4) data trasnfer; and (5) synchronizing clocks so that the data collected by the sensor network is time stamped in a consistent way.

\subsection{Mobility Control for Data Muling}

The algorithm we implemented for locating the first node of the data muling tour starts by positiong the robot in the general area of the network. Given that the general location is known in GPS coordinates, the AUV can perform surface navigation guided by GPS to move toward the node. Once close the AUV descends to the optical communications range. At this point the AUV can perform a spiral search to locate the node. We implemented and tested two methods for the spiral search. Starbug located the nodes by visual servoing to a calibrated color model of the box. This

\footnotetext{
${ }^{8}$ According to the tide tables for Brisbane.
}

method is robust in the presence of drift and currents but requires ambient light. Alternatively, we can use active beaconing without the need for ambient light. Amour located the nodes with active beaconing. During the spiral trajectory guided by magnetic compass, the AUV uses its optical communication system to request the activation of the optical beacon on the Aquafleck. The main disadvantage of this method is greater sensitivity to currents and drift due to the lack of odometry, and the increased power required for the beacon.

Moving from node to node requires information about the relative position of the network. This can be provided as a map input to the AUV. Alternatively, networking can be used to compute the relative coordinates using a method such as our distributed localization algorithm [20] built on top of acoustic ranging. Given the relative location of the new node Starbug located the next node using visual odometry while Amour locates the node using the magnetic compass. Both methods were demonstrated in the pool for maximum travel distances between two adjecent nodes of approximately 5 meters.

Controlling the hover mode is achieved using visual servoing by Starbug, and active beaconing by Amour. Both methods have been demonstrated in the presence of heavy perturbations generated by currents in the ocean and generated manually in the pool.

Data transfer is achieved with a protocol to establish a datalink between the mobile and static node, and a protocol for data transfer. The mobile node begins with a query about the available data. The data is then transmitted in 239 byte checksummed packets. This is a master-slave process with the mobile node taking the role of the master and requesting the data either in a packet by packet mode, or in a groups of packets mode depending on the quality of the communication link. If any packet is lost (for example, when the request does not arrive or data does not arrive) the mobile node times out and sends another request. At the end of the data transmission, the mobile node asks the static node to reset and erase the data that was collected. This simple data transfer protocol relies on state only for the mobile node; the static node is stateless.

A key capability of such a system is the ability to time stamp data collected from different nodes consistently. Because we desire long-term operation for an underwater sensor network (measured in days and weeks) we expect the clocks of the underwater nodes to drift which is why clock synchronization is a very important component of the data muling algorithm. Some studies require no precise timing (for example the rate of change for temperature and pressure data is much slower than the expected clock drift), but for other studies timing is important (for example when monitoring schools of fish or the spread of pollution.) We assume that each static node has its own clock and that clocks tick at different, but constant, rates. The clock of the mobile node is used as reference so that in the beginning of the experiment all the clocks are set to the mobile node's $T_{0}$. When the mobile node uploads the data from a static node it first reads the node's clock $T_{n}$. If this time is different than the current time on the mobile node's clock, all the data time stamps are adjusted to $T_{0}+\left(T_{i}-T_{0}\right) *\left(T_{\text {auv }}-T_{0}\right) /\left(T_{\text {node }}-T_{0}\right)$ and the current time of the node is reset to match the mobile node's time. The alternative solution is to allow the sensor network to coordinate time in a fully distributed way using 


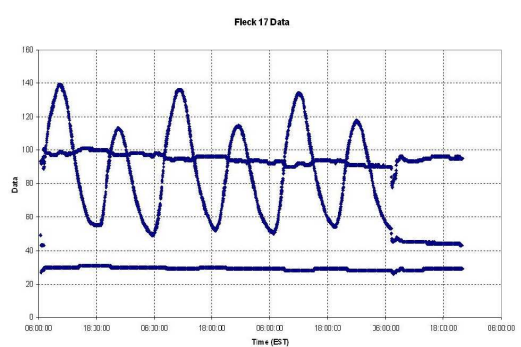

(a)

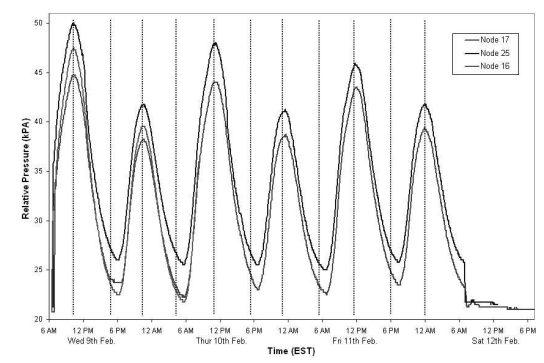

(b)

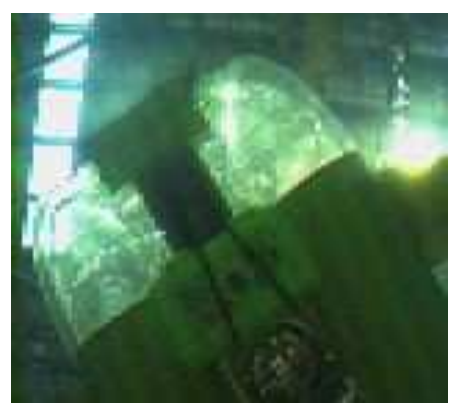

(c)

Figure 5: Data from Aquafleck Deployments. (a) Pressure and temperature data for sensor node 17 deployed in Tingalpa Creek, Brisbane. (b) Pressure data collected by three different sensor nodes. The data was collected for a period of three days (node 16 stopped recording midway through the experiment). The differences in pressure readings are due to differences in the node depth and distances from the ocean. (c) Image of the Starbug AUV taken from a sensor node. This image was collected from the node using optical communication.

a global clock synchronization algorithm. This approach would yield more accurate time stamps but requires a significant number of message broadcasts, which is expensive to do acoustically in water.

\subsection{Integration with AUV systems}

In these experiments Starbug was fitted with an AquaFleck, mounted between the two cylindrical hulls, for optical communications with its sea floor counterpart. We refer to this node as the gateway node. An RS232 serial cable from the box connected to the onboard Linux computer via a waterproof connector on the left rear bulkhead. The gateway node was loaded with the TinyOS GenericBase application. This application accepts messages from the UART that are sent over the optical communication link. It also receives messages from the optical communication link and relays them over the serial link to the Starbug Linux computer. The SerialForwarder application was not used since the cutdown Linux system (flash memory based) has no JVM. Instead we used a C library to implement the protocol.

Communications between an Aquafleck and Starbug was limited by the $57.6 \mathrm{kbaud}$ serial link between the gateway node and the Starbug processor, and the data request protocol overhead of $113 \%$. The measured end-to-end data upload rate between an Aquafleck and Starbug is 2.3 kbyte of stored data per second. The measured end-to-end upload rate between Aquafleck and Amour is 13.1 kbyte of stored data per second. We are using packets with a 293 byte payload.

\subsection{Experimental Results}

We have deployed networks consisting of up to 8 Aquaflecks, Starbug, and Amour at two sites in Brisbane: an $8 \times 5 \times 1.5 \mathrm{~m}$ pool at the CSIRO laboratory, and at an Olympic dive pool at $5 \mathrm{~m}$ depth. The work was conducted during two periods of intense joint experiments: February and August 2005. The Aquaflecks were deployed with an approximate grid topology. Starbug and Amour traversed the network for data muling and to pick up and transport the static sensors.

The sensor network grid is described by parameters loaded by the AUV software at run-time. The parameters are: (a) Total number of nodes; and (b) A list of $\left(x, y, \psi_{o}\right)$ for each node, where $(x, y)$ is the node's location with respect to a world coordinate frame, and $\psi_{o}$ is the magnetic compass deviation at that point. This offset was required for testing in our tank to account for magnetic anomalies due to steel in the floor.

Given an initial starting location the AUV moves toward the first node using visual navigation and compass heading. The down cameras process the color imagery at $5 \mathrm{~Hz}$ to locate the distinctive yellow colored boxes. The front cameras could potentially be used for navigation, to head toward a yellow target in front of the vehicle. However when travelling at $1 \mathrm{~m}$ above the bottom, the field of view of the front cameras restricts visibility to boxes at least $4 \mathrm{~m}$ ahead.

Localization of an AUV underwater is a difficult problem but essential in order to navigate to previously deployed network nodes. In this work the Starbug AUV performs visual odometry using a feature-based structure from motion algorithm $[7,8]$ to maintain a path to the next node despite currents and other disturbances. When the AUV arrives in the vicinity of a node, that node's identity can be used to reset any accumulated localization error.

Once a candidate node has been detected the AUV commences a hover operation and maintains its position with respect to the target. The node is queried (HELLO message) and it responds (ACK message) with its identity, capability, software revision and internal clock value. The identity is compared to the expectation from the navigation process and if a mismatch is found the vehicle will head toward the desired node based on the location it knows it is now at. If no response is detected after a set number of retries then there are two possibilities: we are looking at some other yellowish object or the node has failed, and these two conditions are difficult to distinguish. The shape of the object can provide some information, but given the very wide range of lighting conditions, the shape cannot always be reliably determined. This ambiguous case presents a challenge for resetting the dead-reckoned position estimate: assuming we are at a node when we are not, or assuming we are not at a node when we are, can introduce an error that may not be recoverable.

If the query message confirms the identity of the node we were seeking then the AUV initiates a data transfer dialog. The capability byte has one bit set for each type of sensor present in the node as shown in Table 6. As an example, if a sensor node only had an on-board temperature sensor 


\begin{tabular}{|c|c|}
\hline Bit position & Sensor \\
\hline \hline 0 & On-board Temperature Sensor \\
1 & Water Temperature Sensor \\
2 & Water Pressure Sensor \\
3 & CMU Camera Sensor \\
4 & Acoustic Communication Module \\
\hline
\end{tabular}

Table 6: Sensor Node Capability Array Structure

and a CMU camera sensor, then its capability array would be $1,0,0,1,0$.

The AUV sends a REQUESTDATA message which contains a flag (sensor data or camera data) and a required data index value. The initial value of the index is 0 . The node responds with a NACK if the requested data value is out of range, that is, there is no more data, or with a RAWDATA message. The payload of the RAWDATA message contains the index value, the number of data values and a list of data tuples: (1) Data type ( $0=$ pressure, $1=$ temperature etc), 1 byte. (2) Time stamp, 3 bytes. (3) Data value, 2 bytes. With 6 bytes for each tuple, we can transfer 39 tuples or data points per packet. When the desired data has been received, the AUV increments the index value and requests the next group of data values.

A small number of nodes are also equipped with a camera, and at this stage we are using a CMUCam 2 serially connected to the Fleck at $38.4 \mathrm{kbaud}$. With a resolution of $87 \times 143$ pixels and 3 bytes per pixel (RGB) the total image size is 37323 bytes. The images also have a 2:1 aspect ratio, therefore the effective image size is $174 \times 143$ pixels when displayed. With a resolution of $255 \times 143$ pixels and 1 bytes per pixel (each byte is either red, green, or blue), the total image size is 36465 bytes. The image size exceeds the available RAM by a large amount so we keep the image in the camera and transfer it one row at a time using the camera's virtual window facility. When the AUV sends a TAKEPHOTO message the CMUCam is commanded to capture an image. When a REQUESTDATA message, with the image flag set, is received the appropriate row of the image data is requested from the camera, then sent to the AUV in an IMAGEDATA message. Once again, a low-bandwidth data path, is limiting the overall throughput of our system. A newly developed digital camera daughterboard for the Fleck will alleviate this problem in future.

In two experimental campaigns conducted in February 2005 and August 2005 in the CSIRO laboratory pool and an Olympic dive pool, we have reliably located hundreds of AquaFleck nodes and navigated between them. The Starbug AUV is routinely able to tour all the AquaFlecks in its map. Hundreds of data transfers comprising thousands of packets have been uploaded from our AquaFleck nodes to storage onboard the Starbug AUV.

\section{CONCLUSIONS}

In this paper we reported on a first prototype for an an underwater sensor network we developed, built, and used. We described the hardware, the networking infrastructure, and our experiments with data collection and retrieval.

This work demonstrates that sensor networks are feasible underwater and that data muling provides an effective way to collect, store, and retrieve large volumes of data over long periods of time. We argue that data muling provides a significant power advantage over an acoustic communication network with multihop routing.

Our work shows the benefits of creating underwater systems that have a mix of static and mobile nodes networked together in dual ways, as a combination of acoustic communication for low data rate broadcast and optical communication for high data rate point-to-point communication. The static sensor nodes enable systematic recording of data. The mobile nodes enable efficient data muling and integration, data delivery to a surface base station independent of the physical location of the sensors, and long-term underwater operations of the sensor nodes at fixed locations.

The contributions of this paper include several algorithms for controlling and networking the static and mobile nodes. These algorithms have been instantiated to the specific hardware we developed. However, the algorithms for docking and navigation can be instatiated to other hardware systems with different architecture but similar capabilities. Similarly, the algorithms for optical data encoding and acoustic data encoding and medium access are generic and can be used in future generations of underwater sensors.

Data collection, storage and retrieval in underwater environments is a rich application domain with many technical challenges left to be resolved. We have learned several lessons during the development of this work. Hardware and software reliability are extremely important. Mobility provides an effective and highly power-efficient means for collecting data in sensor networks, for network programming, and more generally speaking for networking the system. However, controlling the mobile nodes in the presence of currents remains challenging and affects the reliability of hovering for data transfers. Above all, underwater sensor networks promise many exciting applications and opportunities to collaborate with marine biologists to enrich our understanding of the underwater world.

\section{Acknowledgements}

This work has been supported in part by CSIRO, MIT's project Oxygen, Intel, Boeing, and NSF awards 0423962, EIA-0202789, IIS-0426838, and ONR's PLUSNet S05-06 project. We are grateful for this support. We thank the entire team at CSIRO for the technical and moral support provided during a period of intense experimentation. Pavan Sikka integrated the optical communication system with the TinyOS stack. Poe Chen for developing the interface between the Flecks and Starbug and for his programming support for data muling. We are also grateful to Leslie Overs for his support with electronics and to Philipp Schmid for his help in building the Aquaflecks and in experimentation. Finally, we thank our reviewers and espeically our shepherd Deborah Estrin for their time and thoughtful suggestions.

\section{REFERENCES}

[1] Autonomous Ocean Sampling Network (AOSN)II, collaborative project. http://www.princeton.edu/d̃dcsl/aosn/.

[2] Whoi modem: http://acomms.whoi.edu/micromodem/.

[3] Akyiditz, I., Pompli, D., And Melodia, T. Underwater sensor networks: Research challenges. Ad-hoc Networks (2005 (to appear)). 
[4] Akyildiz, I. F., Pompili, D., And Melodia, T. Challenges for efficient communication in underwater acoustic sensor networks. ACM Sigbed Review 1, 2 (july 2004).

[5] Bansal, N., And Liu, Z. Capacity, delay and mobility in wireless ad-hoc networks. In INFOCOM (San Francisco, CA, April 2003).

[6] Bokser, V., Oberg, C., Sukhatme, G., And REQuicha, A. A small submarine robot for experiments in underwater sensor networks. In IFAC International Federation of Automatic Control Symposium on Intelligent Autonomous Vehicles (2004).

[7] Dunbabin, M., Roberts, J., Usher, K., Winstanley, G., And Corke, P. A hybrid auv design for shallow water reef navigation. In Proceedings of the 2005 International Conference on Robotics and Automation (Barcelona, Apr. 2005), pp. 2117-2122.

[8] Dunbabin, M., Usher, K., And Corke, P. Visual motion estimation for an autonomous underwater reef monitoring robot. In Proc. International Conference on Field E Service Robotics (Port Douglas, 2005), pp. 57-68.

[9] Fiorelli, E., Leonard, N. E., Bhatta, P., Paley, D., Bachmayer, R., and Fratantoni, D. M. Multi-AUV control and adaptive sampling in Monterey Bay. In Proceedings of the IEEE Autonomous Underwater Vehicles: Workshop on Multiple AUV Operations (Sebasco, ME, USA, June 2004).

[10] Grossglauser, M., And Vetterli, M. Locating nodes with ease: Mobility diffusion of last encounters in ad hoc networks. In INFOCOM (San Francisco, CA, April 2003)

[11] Heidemann, J., Li, Y., Syed, A., Wills, J., And YE, W. Underwater sensor networking: Research challenges and potential applications. Tech. Rep. ISI-TR-2005-603, USC/Information Sciences Institute, July 2005.

[12] Hill, J., Bounadonna, P., And Culler, D. Active message communication for tiny network sensors. In INFOCOM (2001).

[13] Hill, J., Szewczyk, R., Woo, A., Hollar, S., Culler, D., And Pister, K. System architecture directions for network sensors. In ASPLOS (2000).

[14] Howe, B. M., McGinnis, T., And Kirkham, H. Sensor Networks for Cabled Ccean Observatories. EGS - AGU - EUG Joint Assembly, Abstracts from the meeting held in Nice, France, 6 - 11 April 2003, abstract \#12598 (Apr. 2003), 12598-+.

[15] Jain, S., Shah, R., Brunette, W., Borriello, G., AND Roy, S. Exploring mobility for energy efficient data collection in sensor networks. ACM/Kluwer Mobile Networks and Applications Journal to appear (2005), Also in Proc. of 2004 WiOpt 2004.
[16] Jea, D., Somasundra, A., And Srivastava, M. Multiple controlled mobile elements (data mules) for data collection in sensor networks. In IEEE International Conference on Distributed Computing in Sensor Systems (DCOSS) (2005).

[17] Kaiser, W., Pottie, G., Srivastava, M., Sukhatme, G., Villasenor, J., and Estrin, D. Networked infomechanical systems (nims) for ambient intelligence. Technical report 31, UCLA Center for Embedded Networked Systems, 2003.

[18] Kilfoyle, D., And Baggeroer, A. The state of the art in underwater acoustic telemetry. IEEE J. Oceanic Engineering 25, 1 (Jan. 2000), 4-27.

[19] Leonard, J., Bennett, A. A., Smith, C. M., And Feder, H. J. S. Autonomous underwater vehicle navigation. Technical memorandum 98-1, MIT Marine Robotics Laboratory, 1998.

[20] Moore, D., Leonard, J., Rus, D., And Teller, S. Robust distributed network localization with noisy range measurements. In ACM SenSys (Baltimore, MD, Nov. 2004).

[21] Perevalov, E., and Blum, R. Delay limited capacity of ad hoc networks: Asymptotically optimal transmission and relaying strategy. In INFOCOM (San Francisco, CA, April 2003).

[22] Proakis, J., Sozer, E., Rice, J., And Stojanovic, M. Shallow water acoustic networks. IEEE Communications Magazine (Nov. 2001), 114-119.

[23] Schill, F., Zimmer, U., And TrumpF, J. Visible spectrum optical communications and distance sensing for underwater applications. In Proc. Australasian Conf. Robotics and Automation (Canberra, 2004).

[24] Shah, R. C., Roy, S., Jain, S., and Brunette, W. Data mules: Modeling a three-tier architecture for sparse sensor networks. In First IEEE International Workshop on Sensor Network Protocols and Applications (SPNA) (Anchorage, AK, May 2003).

[25] SikkA, P., Corke, P., ANd Overs, L. Wireless sensor devices for animal tracking and control. In Proc. First IEEE Workshop on Embedded Networked Sensors (Tampa, Florida, Nov. 2004).

[26] Tivey, M. A low power, low cost, underwater optical communications system. Ridge 2000 Events (Apr. 2004), 27-29.

[27] Vasilescu, I., Varshavskaya, P., Kotay, K., AND Rus, D. Autonomous modular optical underwater robot (amour): Design, prototype, and feasibility study. In Proceedings of the 2005 International Conference on Robotics and Automation (Barcelona, Apr. 2005).

[28] Ye, W., Heidemann, J., and Estrin, D. An energy-efficient mac protocol for wireless sensor networks. In INFOCOM (New York, NY, June 2002).

[29] YuH, J. Design and control of autonomous underwater robots: A survey. Autonomous Robots 8, 1 (2000), 7-24. 